\title{
New Linear Generalized Synchronization and Parameter Identification between Different Chaotic Systems
}

\author{
Chao Ma ${ }^{1,2, a}$, Xingyuan Wang ${ }^{2}$, Yujuan Tian ${ }^{3}$ \\ ${ }^{1}$ School of Mathematical Sciences, University of Jinan, Jinan, Shandong, 250022, China \\ ${ }^{2}$ School of Electronic and Information Engineering,Dalian University of Technology, Dalian, \\ Liaoning, 116024, China \\ ${ }^{3}$ School of Mathematical Sciences, Shandong Normal University, Jinan, Shandong, 250014, China \\ achaos_ma@163.com
}

Keywords: Stable linear generalized synchronization. Parameter identification. chaotic system.

\begin{abstract}
The stability of a linear generalized synchronization approach between different chaotic systems with unknown parameters is investigated in this paper. An adaptive controller and some parameter update laws are proposed to achieve stable linear generalized synchronization and parameter identification of different chaotic systems. This novel synchronization method effectively unifies complete synchronization, anti-synchronization, projective synchronization, rotation synchronization and function synchronization. The main results are illustrated by two examples: the rotation synchronization between Lorenz and Chen system; the modified projective synchronization between Lorenz system and Lü system.
\end{abstract}

\section{Introduction}

Since the seminal work in [8], chaos synchronization has been a topic of great attention [9, 10], and been applied like dominoes effect in numerous fields including secure communication [11, 12], neurocomputing [13], complex systems [14], human brain and coronary artery [15, 16, 17] and so on. Due to above-mentioned and many potential applications, various different types of chaos synchronization have been developed such as complete synchronization $[8,18,19]$, anti-synchronization [20, 21], phase synchronization [22, 23, 24], lag synchronization [25, 26, 27], generalized synchronization (GS) $[28,29,30,31,32,33,34,35,36,37,38,39,40]$, exponential synchronization [41], impulsive synchronization [42, 43, 44, 45], etc.

Generalized synchronization was first introduced in 1995 [28]. Kocarev and Parlitz gave the mathematical definition of GS in 1996 [29]. The papers [30, 31] proposed an auxiliary and a modified system approach to achieve GS, respectively. Projective synchronization, a subclass of GS, has been developed by some authors. $\mathrm{Li}$ and $\mathrm{Xu}$ considered a projective synchronization in different chaotic systems [32]. A modified projective synchronization of chaotic system was presented in [33]. In Ref. [34], the function projective synchronization of uncertain chaotic systems was given. The projective synchronization of fractional-order hyperchaotic system was achieved in [35]. In [36], lag projective synchronization and anti-synchronization can be achieved under the condition of systems with bounded nonlinearity. Recently, a new definition of GS in nonlinear systems using the concept of differential primitive element is introduced, this new definition, essentially, is a function projective synchronization [37].

Linear synchronization, another subclass of GS, was proposed by Yang and Chua in [38]. In fact, linear synchronization unifies several different types of synchronization such as complete synchronization, anti-synchronization, projective synchronization, modified projective synchronization, rotation synchronization and function projective synchronization. So it is important to design linear synchronization for chaotic systems. Lu and $\mathrm{Xi}$ [39] developed a linear generalized synchronization of continuous-time identical chaotic systems. Hu and $\mathrm{Xu}$ [40] provided a stochastic linear generalized synchronization of chaotic systems based on robust control. However, Yang's method needs strict conditions for the drive system and the linear transformation matrix, and all these linear synchronization 
methods are focus on systems whose parameters are exactly known in advance. But in many practical cases, some system parameters cannot be obtained beforehand.

Motivated by the above discussions, the present paper attempts to provide a new general approach to accomplish linear synchronization of different chaotic systems with unknown parameters. Meanwhile, the unknown parameters of drive system can be identified correctly through parameter update laws. Examples of modified projective synchronization and rotation synchronization are performed to demonstrate the effectiveness of the approach.

\section{The adaptive approach for generalized linear synchronization}

The chaotic dynamics of the drive system

$$
\dot{x}=f(x)+D(x) \Theta
$$

and the response system

$$
\dot{\boldsymbol{y}}=\boldsymbol{B} \boldsymbol{y}+\boldsymbol{g}(\boldsymbol{y})+\boldsymbol{U}(\boldsymbol{x}, \boldsymbol{y})
$$

where $\boldsymbol{x}=\left(x_{1}, x_{2}, \ldots, x_{n}\right)^{T}, \boldsymbol{y}=\left(y_{1}, y_{2}, \ldots, y_{n}\right)^{T} \in \boldsymbol{R}^{n}$ are state vectors of the systems (1) and (2). $\boldsymbol{\Theta}=\left(\theta_{1}, \theta_{2}, \ldots, \theta_{k}\right)^{T} \in \boldsymbol{R}^{k}(k \leq n)$ is the unknown parameter of the drive system (1). $\boldsymbol{D}(\boldsymbol{x})$ : $\boldsymbol{R}^{n} \rightarrow \boldsymbol{R}^{n \times k}$ is the coefficient vector of the unknown parameter. $\boldsymbol{f}(\boldsymbol{x})$ is independent of the unknown parameters. $\boldsymbol{B} \boldsymbol{y} \in \boldsymbol{R}^{n}$ and $\boldsymbol{g}(\boldsymbol{y})$ are the linear and nonlinear part of system (2), respectively. $\boldsymbol{U}(\boldsymbol{x}, \boldsymbol{y})=\left\{u_{1}, u_{2}, \ldots, u_{n}\right\}^{T} \in \boldsymbol{R}^{n}$ is the controller to be designed.

Remark. System (1) includes many well known systems, such as the Lorenz system family, Chua's circuit, Duffing system, Rösler system, some hyper-chaotic systems, etc.

Definition 1. The two different systems (1) and (2) are said to be in a state of linear generalized synchronization, if there exists a linear transformation matrix $\boldsymbol{H}=\left(h_{i, j}\right)_{n \times n}\left(h_{i, j}\right.$ are not all zero constants), a manifold $\boldsymbol{M}=\left\{(\boldsymbol{x}(t), \boldsymbol{y}(t) \mid \boldsymbol{y}(t)=-\boldsymbol{H} \boldsymbol{x}(t)\}\right.$, and a set $\boldsymbol{\Omega} \subset \boldsymbol{R}^{n} \times \boldsymbol{R}^{n}$ with $\boldsymbol{M} \subset \boldsymbol{\Omega}$ such that all trajectories $(\boldsymbol{x}(t), \boldsymbol{y}(t))$ of (1) and (2) with any initial conditions in $\Omega$ approach $\boldsymbol{M}$ as time $t$ goes to infinity, that is to say,

$$
\lim _{t \rightarrow \infty}\|\boldsymbol{e}(t)\|=\lim _{t \rightarrow \infty}\|\boldsymbol{y}(t)+\boldsymbol{H} \boldsymbol{x}(t)\|=\mathbf{0}
$$

where $\boldsymbol{e}(t)=\boldsymbol{y}(t)+\boldsymbol{H} \boldsymbol{x}(t)$ is the synchronization error. The above equation implies $\boldsymbol{e}(t)$ is globally asymptotically stable.

Remark. In definition 1, if we take the manifold $\boldsymbol{M}$ as $\{(\boldsymbol{x}(t), \boldsymbol{y}(t) \mid \boldsymbol{y}(t)=\boldsymbol{H} \boldsymbol{x}(t)\}$, the synchronization error will be $\boldsymbol{e}(t)=\boldsymbol{y}(t)-\boldsymbol{H} \boldsymbol{x}(t)$.

Theorem 2. For drive system (1), response system (2) and any linear transformation matrix $\boldsymbol{H}$, if the controller and the parameter update laws are given by

$$
U(x, y)=B H x-g(y)-H f(x)-H D(x) \Theta^{*}+\Delta e
$$

and

$$
\dot{\boldsymbol{\Theta}}^{*}=(\boldsymbol{H} \boldsymbol{D}(\boldsymbol{x}))^{T} \boldsymbol{e}
$$

where $\Theta^{*}=\left(\theta_{1}^{*}, \theta_{2}^{*}, \ldots, \theta_{k}^{*}\right)^{T}$ is the estimate of the unknown parameter $\boldsymbol{\Theta}, \boldsymbol{\Delta}$ is a constant matrix satisfying $\boldsymbol{B}+\boldsymbol{\Delta}<0$ (negative definite). Then the linear generalized synchronization of systems (2) and (1) will be stability and the uncertain parameters $\Theta$ will be determined in the process of synchronization. 
proof: From the drive system (1) and response system (2), the error dynamics can be expressed as

$$
\dot{\boldsymbol{e}}=\dot{\boldsymbol{y}}(t)+\boldsymbol{H} \dot{\boldsymbol{x}}(t)=\boldsymbol{B} \boldsymbol{y}+\boldsymbol{g}(\boldsymbol{y})+\boldsymbol{U}(\boldsymbol{x}, \boldsymbol{y})+\boldsymbol{H} \boldsymbol{f}(\boldsymbol{x})+\boldsymbol{H} \boldsymbol{D}(\boldsymbol{x}) \Theta
$$

Applying controller (3) and parameter update laws (4) to (9) yields the new error dynamics:

$$
\dot{e}=B e+H D(x) \bar{\Theta}+\Delta e=(B+\Delta) e+H D(x) \bar{\Theta}
$$

where $\bar{\Theta}=\Theta-\Theta^{*}$ is the parameter estimate error.

Consider the following Lyapunov function:

$$
\boldsymbol{V}(\boldsymbol{e}, \overline{\boldsymbol{\Theta}})=\frac{1}{2} \boldsymbol{e}^{T} \boldsymbol{e}+\frac{1}{2} \overline{\boldsymbol{\Theta}}^{T} \overline{\boldsymbol{\Theta}}
$$

Differentiating the Lyapunov function $\boldsymbol{V}(\boldsymbol{e}, \overline{\boldsymbol{\Theta}})$ along the trajectories of the error dynamics (10) yields

$$
\begin{aligned}
\dot{\boldsymbol{V}}(\boldsymbol{e}, \overline{\boldsymbol{\Theta}}) & =\boldsymbol{e}^{T} \dot{\boldsymbol{e}}+\overline{\boldsymbol{\Theta}}^{T} \dot{\overline{\boldsymbol{\Theta}}} \\
& =\boldsymbol{e}^{T}(\boldsymbol{B}+\boldsymbol{\Delta}) \boldsymbol{e}+\boldsymbol{e}^{T} \boldsymbol{H} \boldsymbol{D}(\boldsymbol{x}) \overline{\boldsymbol{\Theta}}-\overline{\boldsymbol{\Theta}}^{T}(\boldsymbol{H} \boldsymbol{D}(\boldsymbol{x}))^{T} \boldsymbol{e} \\
& =\boldsymbol{e}^{T}(\boldsymbol{B}+\boldsymbol{\Delta}) \boldsymbol{e}
\end{aligned}
$$

for any constant matrix $\boldsymbol{B}$, there is always matrix $\boldsymbol{\Delta}$ such that $\boldsymbol{B}+\boldsymbol{\Delta}<0$. That is to say, with a reasonable choice of $\Delta$, the following inequality can be satisfied

$$
\dot{\boldsymbol{V}}(\boldsymbol{e}, \overline{\boldsymbol{\Theta}})<0
$$

On the basis of the Lyapunov stability theorem and LaSalle's theorem, the zero point of the error dynamics (10) is globally asymptotically stable, i.e, $\lim _{t \rightarrow \infty}\|\boldsymbol{e}(t)\|=\mathbf{0}$. According to (10), one can obtain that $\boldsymbol{H} \boldsymbol{D}(\boldsymbol{x}) \overline{\boldsymbol{\Theta}}=\mathbf{0}$ as time t goes to infinity. Because $\boldsymbol{H} \neq 0, \boldsymbol{D}(\boldsymbol{x})$ is non-zero and bounded, $\bar{\Theta}$ goes to zero as $t \rightarrow \infty$, which means that $\lim _{t \rightarrow \infty} \Theta^{*}=\Theta$.

Hence, all trajectories $(\boldsymbol{x}(t), \boldsymbol{y}(t))$ of (1) and (2) starting with any initial conditions approach $\boldsymbol{M}=\left\{\boldsymbol{e} \in \boldsymbol{R}, \overline{\boldsymbol{\Theta}} \in \boldsymbol{R}^{k} \mid \boldsymbol{e}=\mathbf{0}, \overline{\boldsymbol{\Theta}}=\mathbf{0}\right\}$ as time $t$ goes to infinity. So the globally asymptotically linear generalized synchronization of two chaotic systems is stability. Meanwhile, the parameter identification problem is solved. This completes the proof of Theorem 1.

proof: From the drive system (1) and response system (2), the error dynamics can be expressed as

$$
\dot{\boldsymbol{e}}=\dot{\boldsymbol{y}}(t)+\boldsymbol{H} \dot{\boldsymbol{x}}(t)=\boldsymbol{B} \boldsymbol{y}+\boldsymbol{g}(\boldsymbol{y})+\boldsymbol{U}(\boldsymbol{x}, \boldsymbol{y})+\boldsymbol{H} \boldsymbol{f}(\boldsymbol{x})+\boldsymbol{H} \boldsymbol{D}(\boldsymbol{x}) \Theta
$$

Applying controller (3) and parameter update laws (4) to (9) yields the new error dynamics:

$$
\dot{e}=B e+H D(x) \bar{\Theta}+\Delta e=(B+\Delta) e+H D(x) \bar{\Theta}
$$

where $\bar{\Theta}=\Theta-\Theta^{*}$ is the parameter estimate error.

Consider the following Lyapunov function:

$$
\boldsymbol{V}(\boldsymbol{e}, \overline{\boldsymbol{\Theta}})=\frac{1}{2} \boldsymbol{e}^{T} \boldsymbol{e}+\frac{1}{2} \overline{\boldsymbol{\Theta}}^{T} \overline{\boldsymbol{\Theta}}
$$
yields

Differentiating the Lyapunov function $\boldsymbol{V}(\boldsymbol{e}, \overline{\boldsymbol{\Theta}})$ along the trajectories of the error dynamics (10)

$$
\begin{aligned}
\dot{\boldsymbol{V}}(\boldsymbol{e}, \overline{\boldsymbol{\Theta}}) & =\boldsymbol{e}^{T} \dot{\boldsymbol{e}}+\overline{\boldsymbol{\Theta}}^{T} \dot{\overline{\boldsymbol{\Theta}}} \\
& =\boldsymbol{e}^{T}(\boldsymbol{B}+\boldsymbol{\Delta}) \boldsymbol{e}+\boldsymbol{e}^{T} \boldsymbol{H} \boldsymbol{D}(\boldsymbol{x}) \overline{\boldsymbol{\Theta}}-\overline{\boldsymbol{\Theta}}^{T}(\boldsymbol{H} \boldsymbol{D}(\boldsymbol{x}))^{T} \boldsymbol{e} \\
& =\boldsymbol{e}^{T}(\boldsymbol{B}+\boldsymbol{\Delta}) \boldsymbol{e}
\end{aligned}
$$


for any constant matrix $\boldsymbol{B}$, there is always matrix $\boldsymbol{\Delta}$ such that $\boldsymbol{B}+\boldsymbol{\Delta}<0$. That is to say, with a reasonable choice of $\Delta$, the following inequality can be satisfied

$$
\dot{\boldsymbol{V}}(\boldsymbol{e}, \overline{\boldsymbol{\Theta}})<0
$$

On the basis of the Lyapunov stability theorem and LaSalle's theorem, the zero point of the error dynamics (10) is globally asymptotically stable, i.e, $\lim _{t \rightarrow \infty}\|\boldsymbol{e}(t)\|=\mathbf{0}$. According to (10), one can obtain that $\boldsymbol{H} \boldsymbol{D}(\boldsymbol{x}) \overline{\boldsymbol{\Theta}}=\mathbf{0}$ as time t goes to infinity. Because $\boldsymbol{H} \neq 0, \boldsymbol{D}(\boldsymbol{x})$ is non-zero and bounded, $\bar{\Theta}$ goes to zero as $t \rightarrow \infty$, which means that $\lim _{t \rightarrow \infty} \Theta^{*}=\Theta$.

Hence, all trajectories $(\boldsymbol{x}(t), \boldsymbol{y}(t))$ of (1) and (2) starting with any initial conditions approach $\boldsymbol{M}=\left\{\boldsymbol{e} \in \boldsymbol{R}, \overline{\boldsymbol{\Theta}} \in \boldsymbol{R}^{k} \mid \boldsymbol{e}=\mathbf{0}, \overline{\boldsymbol{\Theta}}=\mathbf{0}\right\}$ as time $t$ goes to infinity. So the globally asymptotically linear generalized synchronization of two chaotic systems is stability. Meanwhile, the parameter identification problem is solved. This completes the proof of Theorem 1.

Remark. In the more general case $\boldsymbol{y}=-\boldsymbol{H} \boldsymbol{x}(t)-\boldsymbol{P}$ ( $\boldsymbol{P}$ is a non-zero constant vector), one need to subtract only $\boldsymbol{P}$ from the right side of (3).

Remark. Many synchronization phenomenons are the special case of the problem considered in this paper, such as complete synchronization $(\boldsymbol{H}=-\boldsymbol{I})$, anti-synchronization $(\boldsymbol{H}=\boldsymbol{I})$, projective synchronization $(\boldsymbol{H}=\alpha \boldsymbol{I})$, modified projective synchronization $\left(\boldsymbol{H}=\operatorname{diag}\left(\gamma_{1}, \gamma_{2}, \ldots, \gamma_{n}\right)\right)$, rotation synchronization $\left(\boldsymbol{H}=\left(\begin{array}{ccc}-\cos \phi & \sin \phi & 0 \\ -\sin \phi & -\cos \phi & 0 \\ 0 & 0 & -1\end{array}\right)\right.$, the response system will be synchronized with the trajectory obtained by rotating (angle $\phi$ ) the drive system around the $z$ axis).

\section{Application examples}

Two simulation examples of linear generalized synchronization are considered to illustrate the the effectiveness of the approach described in Section 2.

Simulation 1: We consider the rotation synchronization of Lorenz system and Chen's system. The drive and response systems are given by the following equations. For the drive system (Lorenz)

$$
\left\{\begin{array}{l}
\dot{x}_{1}=\theta_{1}\left(x_{2}-x_{1}\right) \\
\dot{x}_{2}=\theta_{2} x_{1}-x_{1} x_{3}-x_{2} \\
\dot{x}_{3}=x_{1} x_{2}-\theta_{3} x_{3}
\end{array}\right.
$$

where $x_{1}, x_{2}, x_{3}$ are state vectors, and $\theta_{1}, \theta_{2}, \theta_{3}$ are unknown parameters to be identified. When $\theta_{1}=10, \theta_{2}=28$, and $\theta_{3}=8 / 3$, the system (13) has a chaotic attractor.

The response system (Chen) is given as below

$$
\left\{\begin{array}{l}
\dot{y}_{1}=35\left(y_{2}-y_{1}\right)+u_{1} \\
\dot{y}_{2}=28 y_{2}-7 y_{1}-y_{1} y_{3}+u_{2} \\
\dot{y}_{3}=y_{1} y_{2}-3 y_{3}+u_{3}
\end{array}\right.
$$

where $y_{1}, y_{2}, y_{3}$ are state vectors, and $u_{1}, u_{2}, u_{3}$ are controllers.

According to the synchronization scheme proposed in the previous section, one can obtain that

$$
\boldsymbol{f}(\boldsymbol{x})=\left(\begin{array}{c}
0 \\
-x_{1} x_{3}-x_{2} \\
x_{1} x_{2}
\end{array}\right), \boldsymbol{D}(\boldsymbol{x})=\left(\begin{array}{ccc}
x_{2}-x_{1} & 0 & 0 \\
0 & x_{1} & 0 \\
0 & 0 & -x_{3}
\end{array}\right),
$$




$$
\boldsymbol{B}=\left(\begin{array}{ccc}
-35 & 35 & 0 \\
-7 & 28 & 0 \\
0 & 0 & -3
\end{array}\right), \boldsymbol{g}(\boldsymbol{x})=\left(\begin{array}{c}
0 \\
-y_{1} y_{3} \\
y_{1} y_{2}
\end{array}\right)
$$

Here, we choose $\boldsymbol{H}=\left(\begin{array}{ccc}-\cos \phi & \sin \phi & 0 \\ -\sin \phi & -\cos \phi & 0 \\ 0 & 0 & -1\end{array}\right), \phi=\pi / 4$, and $\boldsymbol{\Delta}=\left(\begin{array}{ccc}0 & -35 & 0 \\ 7 & -39 & 0 \\ 0 & 0 & 0\end{array}\right)$. According to (3) and (4), one can obtain the controller as follows

$$
\left\{\begin{array}{l}
u_{1}=-69 \lambda x_{2}+\lambda x_{1} x_{3}+\lambda\left(x_{2}-x_{1}\right) \theta_{1}^{*}-\lambda x_{1} \theta_{2}^{*}-35 e^{2} \\
u_{2}=-21 \lambda x_{1}-36 \lambda x_{2}+y_{1} y_{3}-\lambda x_{1} x_{3}+\lambda\left(x_{2}-x_{1}\right) \theta_{1}^{*}+\lambda x_{1} \theta_{2}^{*}+7 e_{1}-39 e_{2} \\
u_{3}=3 x_{3}-y_{1} y_{2}+x_{1} x_{2}-x_{3} \theta_{3}^{*}
\end{array}\right.
$$

and the parameter update laws

$$
\left\{\begin{aligned}
\dot{\theta}_{1}^{*} & =-\lambda\left(x_{2}-x_{1}\right)\left(e_{1}+e_{2}\right) \\
\dot{\theta}_{2}^{*} & =\lambda x_{1}\left(e_{1}-e_{2}\right) \\
\dot{\theta}_{3}^{*} & =x_{3} e_{3}
\end{aligned}\right.
$$

where $\lambda=\sqrt{2} / 2$.

The true value of unknown parameters are chosen as $\theta_{1}=10, \theta_{2}=28, \theta_{3}=8 / 3$, and the corresponding initial conditions are $\boldsymbol{x}(0)=(2,3,7)^{T}, \boldsymbol{y}(0)=(3,-3.5,-1)^{T}, \boldsymbol{\Theta}^{*}=(8,10,5)^{T}$. To show the effectiveness of the proposed method, the controller is activated at $5 \mathrm{sec}$. Figure 1.(a) displays the projection of the synchronized attractors onto the plane $x_{1}-x_{2}\left(y_{1}-y_{2}\right)$. Figure 1.(b) shows the time evolution of the synchronization errors $\boldsymbol{E}=\left(e_{1}, e_{2}, e_{3}\right)^{T} \rightarrow \mathbf{0}$ as $t \rightarrow \infty$, which means that the response system is synchronized with the drive system. Figure 1.(c) illustrates the estimate parameters $\mathbf{\Theta}^{*}=\left(\theta_{1}, \theta_{2}, \theta_{3}\right)^{T}$ converge to their true values.
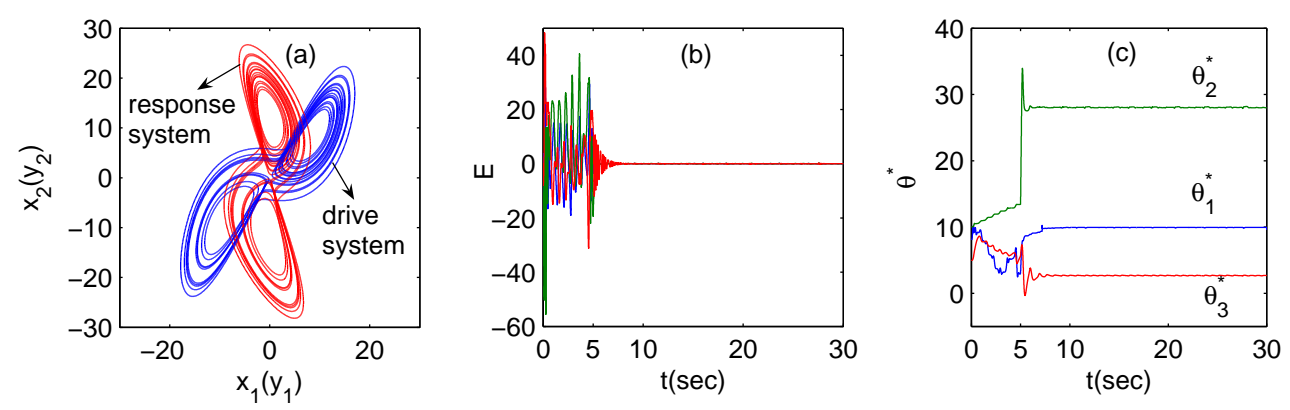

Fig. 1: (color online) The rotation synchronization of Lorenz system and Chen's system.

Simulation 2: We consider the modified projective synchronization of Lorenz (drive) system and Lü (response) system. The Lü system with controller is expressed as

$$
\left\{\begin{array}{l}
\dot{y}_{1}=36\left(y_{2}-y_{1}\right)+u_{1} \\
\dot{y}_{2}=20 y_{2}-y_{1} y_{3}+u_{2} \\
\dot{y}_{3}=y_{1} y_{2}-3 y_{3}+u_{3}
\end{array}\right.
$$

The true value of unknown parameters in drive system are also chosen as $\theta_{1}=10, \theta_{2}=28$, $\theta_{3}=8 / 3$. The control input is also activated at $5 \mathrm{sec}$. For any set of initial conditions, the projections of the synchronized attractors onto the plane $x_{1}-x_{2}\left(y_{1}-y_{2}\right)$ and $x_{1}-x_{3}\left(y_{1}-y_{3}\right)$ are shown in Figure 2.(a) and Figure 2.(b), respectively. Figure 2.(c) is the evolution of the synchronization error $\boldsymbol{E}$. Figure 2.(d) is the estimate parameters. 

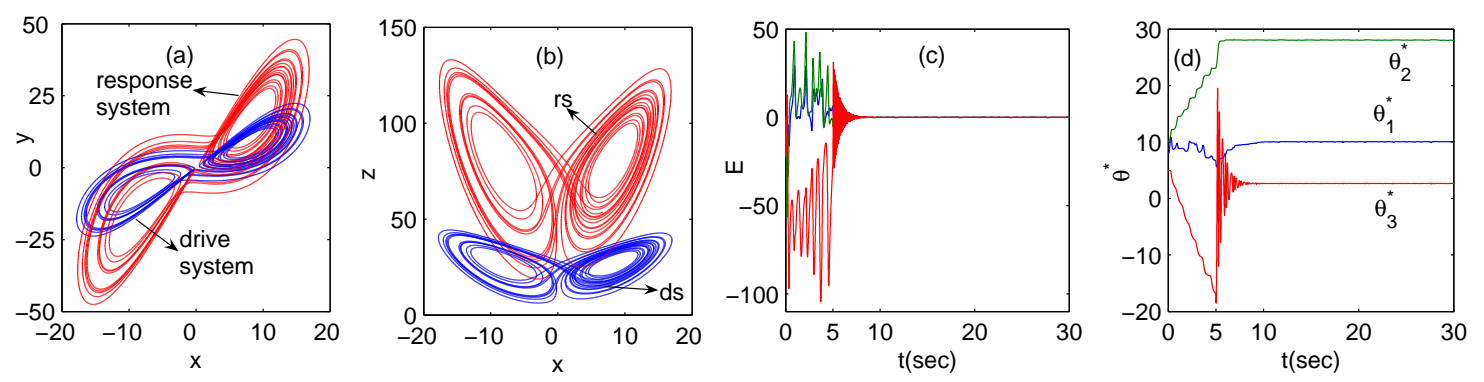

Fig. 2: (color online) The modified projective synchronization of Lorenz system and Lü system.

\section{Conclusions}

In this paper, we present a method to design a response system to recognize linear generalized synchronization with the drive system with unknown parameters, and the stability of this synchronization is proved. Through this method, we not only achieve the linear synchronization of two different chaotic systems, but also estimate the unknown parameters. The modified projective synchronization and rotation synchronization of Lorenz system family are chosen to illustrate the effectiveness of the proposed method.

\section{Acknowledgments}

This research is partially supported by the National Natural Science Foundation of China (61173183, 11501333 ), the Fundamental Research Funds for the Central Universities (DUT12JB06), the Tianyuan Special Funds of the National Natural Science Foundation of China (11426146), the Promotive Research Fund for Excellent Young and Middle-Aged Scientists of Shandong Province (BS2012SF026), National Natural Science Foundation of Shandong Province (ZR2015AL015), The Doctoral Fund of University of Jinan (XBS1337).

\section{References}

[1] Dj.M. Maric, P.F. Meier and S.K. Estreicher: Mater. Sci. Forum Vol. $83-87$ (1992), p. 119

[2] M.A. Green: High Efficiency Silicon Solar Cells (Trans Tech Publications, Switzerland 1987).

[3] Y. Mishing, in: Diffusion Processes in Advanced Technological Materials, edtied by D. Gupta Noyes Publications/William Andrew Publising, CityplaceNorwich, StateNY (2004), in press.

[4] G. Henkelman, G.Johannesson and H. J $\square$ nsson, in: Theoretical Methods in Condencsed Phase Chemistry, edited by S.D. Schwartz, volume 5 of Progress in Theoretical Chemistry and Physics, chapter, 10, Kluwer Academic Publishers (2000).

[5] R.J. Ong, J.T. Dawley and P.G. Clem: submitted to Journal of Materials Research (2003)

[6] P.G. Clem, M. Rodriguez, J.A. Voigt and C.S. CityplaceAshley, country-regionU.S. Patent 6,231,666. (2001)

[7] Information on http://www.weld.labs.gov.cn

[8] L.M. Pecora and T.L. Carroll: Phys. Rev. Lett Vol. 64 (1990), p. 821

[9] X.Y. Wang: Chaos in complex nonlinear systems(Electronic Industry Press, Beijing 2003). 
[10] S. Boccaletti, J. Kurths, G. Osipov, et al: Phys. Rep Vol. 366 (2002), p. 1

[11] A. Argyris, D. Syvridis, L. Larger, et al: Nature Vol. 438 (2005), p. 343

[12] O.I. Moskalenko, A.A. Koronovskii and A.E. Hramov: Phys. Lett. A Vol. 374 (2010), p. 2925

[13] F.C. Hoppensteadt and E. Izhikevich: Oscillatary neurocomputers with dynamic connectivity, country-regionU.S. Patent 6,957,204. (2005)

[14] R. Yamapi, G. Filatrella, M.A. Aziz-Alaoui and H. Kadji: Abst. Appl. Anal (2014), 953813.

[15] E. Rodriguez, N. George et al: Nature Vol. 397 (1999), p. 430

[16] S.K. Dana, P.K. Roy and J. Kurths: Complex dynamics in physiological systems: From heart to $\operatorname{brain}$ (Springer, New York 2009)

[17] C.C. Wang and H.T. Yau: Abst. Appl. Anal (2013), 209718

[18] W. Lin and Y.B. He: Chaos Vol. 15 (2005), 023705

[19] A.S. Landsman and I.B. Schwartz: Phys. Rev. E Vol. 75 (2007), 026201

[20] C.M. Kim, S.W. Rim, w.H. Key, et al: Phys. Lett. A Vol. 320 (2003), p. 39

[21] C. Ma, X.Y. Wang and Y.J. Tian: Chin. J. Phys Vol. 49 (2011), p. 1273

[22] M.G. Rosenblum, A.S. Pikovsky and J. Kurths: Phys. Rev. Lett Vol. 76 (1996), p. 1804

[23] E.R. Rosa, E. Ott and M.H. Hess: Phys. Rev. Lett Vol. 80 (1998), p. 1642

[24] D.V. Senthilkumar, M. Lakshmanan and J. Kurths: Phys. Rev., E Vol. 74 (2006), 035205

[25] M.G. Rosenblum, A.S. Pikovsky and J. Kurths: Phys. Rev. Lett Vol. 78 (1997), p. 4193

[26] Y.H. Sun and J.D. Cao: Phys. Lett. A Vol. 364 (2007), p. 277

[27] C. Hu, J. Yu, H.J. Jiang and Z.D. Teng: Chaos Vol. 20 (2010), 023108

[28] N.F. Rulkov, M.M. ushchik and L.S. Tsimring: Phys. Rev. E Vol. 51 (1995), p. 980

[29] L. Kocarev and U. Parlitz: Phys. Rev. Lett Vol. 76 (1996), p. 1816

[30] H.D.I. Abarbanel adn N.F. Rulkov: Phys. Rev. E Vol. 53 (1996), p. 4528

[31] A.E. Hramov adn A.A. Koronovskii: Phys. Rev. E Vol. 71 (2005), 067201

[32] R.H. Li, W. Xu and S. Li: Phys. Lett. A Vol. 367 (2007), p. 199

[33] X.Y. Wang, X.P. Zhang and C. Ma: Nonlin. Dynam Vol. 69 (2012), p. 511

[34] Y.G. Yu and H.X. Li: Nonlin. Anal. RWA Vol. 11 (2010), p. 2456

[35] L. Liu, D.L. Liang and C.X. Liu: Nonlin. Dynam Vol. 69 (2012), p. 1929

[36] T.B. Wang, S.W. Zhao and W.N. Zhou: Adv. Diff. Equat (2013) DOI: 10.1186/1687-1847-2013225

[37] R. Martinez-Guerra and Mata-Machuca: Appl. Math. Comput Vol. 232 (2014), p. 848 
[38] T. Yang and L.O. Chua: Int. J. Bifurcat. Chaos Vol. 9 (1999), p. 215

[39] J.G. Lu and Y.G. Xi: Chaos. Solit. Fract Vol. 17 (2003), p. 825

[40] A.H. Hu and Z.Y. Xu: Phys. Lett. A Vol.372 (2008), p. 3814

[41] H.G. Zhang, T.D. Ma, G.B. Huang and Z.L. Wang: IEEE Trans. System Man Cybernetics B Vol. 40 (2010), p. 831

[42] C.D. Li, X.F. Liao and X.Y.Zhang: Chaos Vol. 15 (2005), 023104

[43] H.G. Zhang, T.D. Ma, W. Yu and J. Fu: Chin. Phys. B Vol. 17 (2008), p. 3616

[44] C. Ma and X.Y. Wang: Nonlin. Dynam Vol.70 (2012), p. 551

[45] G.Z. Feng and J.D. Cao: Adv. Diff. Equat (2013) DOI: 10.1186/1687-1847-2013-24 